\title{
Gut Microbiome Influences The Neuroinflammatory Response To Traumatic Brain Injury
}

\section{Akshita Jade Kumar}

University of Texas McGovern Medical School: The University of Texas Health Science Center at Houston John P and Katherine G McGovern Medical School https://orcid.org/0000-0002-4021-4092

\section{Supinder Singh Bedi ( $\nabla$ supinder.bedi@uth.tmc.edu )}

University of Texas Health Science Center at Houston https://orcid.org/0000-0003-1938-1790

\section{Naama Toledano-Furman}

University of Texas McGovern Medical School: The University of Texas Health Science Center at Houston John P and Katherine G McGovern Medical School

\section{Louis Carrillo}

University of Texas McGovern Medical School: The University of Texas Health Science Center at Houston John P and Katherine G McGovern Medical School

\section{Fanni Cardenas}

University of Texas McGovern Medical School: The University of Texas Health Science Center at Houston John P and Katherine G McGovern Medical School

\section{Henry Caplan}

University of Texas McGovern Medical School: The University of Texas Health Science Center at Houston John P and Katherine G McGovern Medical School

\section{David Sequeira}

University of Texas McGovern Medical School: The University of Texas Health Science Center at Houston John P and Katherine G McGovern Medical School

\section{Allison L Speer}

University of Texas McGovern Medical School: The University of Texas Health Science Center at Houston John P and Katherine G McGovern Medical School

\section{Scott D Olson}

University of Texas McGovern Medical School: The University of Texas Health Science Center at Houston John P and Katherine G McGovern Medical School

\section{Charles E. Wade}

University of Texas McGovern Medical School: The University of Texas Health Science Center at Houston John P and Katherine G McGovern Medical School

\section{Charles S. Cox}

University of Texas McGovern Medical School: The University of Texas Health Science Center at Houston John P and Katherine G McGovern Medical School 
Research

Keywords: traumatic brain injury, microglia, microbiome, gut-brain axis, immunology

Posted Date: July 14th, 2021

DOI: https://doi.org/10.21203/rs.3.rs-665023/v1

License: (c) (i) This work is licensed under a Creative Commons Attribution 4.0 International License. Read Full License 


\section{Abstract}

Background: Traumatic brain injury $(\mathrm{TBI})$ is a systemic injury that disrupts a complex arrangement of interacting cells in the brain and in the gastrointestinal tract (GI). Disruption in the brain results in neuroinflammation, in which microglia are a central component along with cytokines and other soluble factors [pro and anti-inflammatory microglia (M1:M2)]. Disruption in the GI due to TBI results in a systemic inflammation which is dependent upon the gut microbiome (GM). Gut microbiome can influence microglia in the brain via the gut-brain axis. In order to determine if the microbiome-microglia connections via the gut-brain axis can be modulated, we used probiotics and antibiotics in a rodent TBI model to evaluate the microbiome-microglial connections in acute and chronic experiments.

Methods: The temporal effects of treatment (probiotics or antibiotics) were used to evaluate the gutassociated lymphoid tissue (GALT) influence on the microglial response at 72 hours or 21 days after a cortical contusion injury $(\mathrm{CCl})$, a rodent model of TBI. Injured animals received daily probiotics, antibiotics, or no treatment. Sham-injured animals (controls) did not receive any treatment.

Results: Twenty-one days of probiotic treatment attenuated the pro-inflammatory response of microglia (M1:M2) after CCI. The post-injury inflammatory response was heightened in the GALT with antibioticinduced dysbiosis which resulted in amplification of the pro-inflammatory microglial response.

Conclusions: Probiotic treatment after TBI is a potential therapeutic in attenuating microglial activation through anti-inflammatory signaling.

\section{Background}

Microglia are the resident immune cells of the brain. Under homeostasis, microglia are highly mobile and provide continuous surveillance of their cellular milieu. Microglia have other functions such as promoting learning dependent synapse formation, axonal regeneration, and removal of defunct axon terminals (1). Microglial development and functions are also influenced by the gut microbiome (GM) via the gut-brain axis (1).

Traumatic Brain Injury (TBI) is a systemic injury that effects the entire body. TBI causes a loss of neurons, rearrangement of synaptic connections and subsequent microglia-dependent chronic neuroinflammation. Neuroinflammation is a complex interaction between the cellular (neurons, astrocytes and microglia) and soluble components (cytokines and chemokines) of the central nervous system (CNS). When activated after an injury, microglia undergoing considerable remodeling. They retract their processes and typically adopt an amoeboid morphology (2-4). Microglia are characterized based on their phenotype, which changes in response to environmental stimuli secondary to physiological stress or trauma. Once activated, microglia express surface markers that correlate with neurotoxicity and a pro-inflammatory state or a pro-repair phenotype and an anti-inflammatory state (5). Acutely after injury, microglia polarize towards an pro-inflammatory phenotype, leading to further neurotoxicity and cellular damage due to the disruption of cellular homeostasis $(6,7)$. 
Traumatic brain injury also results in significant systemic manifestations resulting in the dysfunction of non-neurological organ systems. TBI also disrupts the complex arrangement of interacting cells in the gastrointestinal tract (GI). Disruption in the $\mathrm{GI}$ due to TBI results in a systemic inflammation which is dependent upon the GM (8). It has been demonstrated that gastrointestinal dysfunction, or dysbiosis, induces a pro-inflammatory reaction and exacerbates lesion volume and functional deficits in response to ischemic stroke in a rodent model (9). Gut dysfunction can result in ileus, mucosal abnormalities, and disrupted gut permeability, all of which can amplify the systemic inflammatory response and negatively affect clinical outcomes after injury $(10,11)$. Early enteral nutritional support correlates with improved outcomes after TBI, highlighting the complex connection between the gut and brain $(12,13)$. Recent patient data indicate that chronic TBI results in altered fecal microbiome years after the injury (14). The gut-associated lymphoid tissue (GALT) is comprised of the Peyer's patches (PP) and the mesenteric lymph nodes (MLN). The PPs are lymphoid follicles scattered throughout the intestinal tract and serve to mount a protective immune response while continuously surveying the gut-microbe environment. They play a critical role between innate and adaptive immunity through the regulation of T-cell differentiation after exposure to foreign antigens (15). From the PPs, cellular signals drain into the MLNs, which then mount a systemic response in order to maintain homeostasis during physiological stress (16). The MLNs play a significant role in T-cell activation in the gastrointestinal system. Dendritic cells and macrophages (CD11 + cells) surveying the gut continually drain into the MLNs in order to influence development and migration of the adaptive immune system through T-cell activation (17).

We hypothesized that manipulating the gut microbiome via the gut-brain axis through the use of use of probiotics and antibiotics decreases the microglial-dependent neuroinflammation. We evaluated the gutassociated lymphoid tissue influence on the CNS inflammatory response following traumatic brain injury in a rodent TBI model.

\section{Methods}

\section{Animals}

Male Sprague Dawley rodents were obtained from Envigo (Houston, TX) and weighed between 250-350g at the time of all experiments. Gut bacteria can cross colonize between animals in the same cage. To prevent such cross-contamination animals were singly housed ( 1 per cage). Animals were granted ad libitum access to food and water that had been irradiated beforehand to limit contamination. Housing was confided in the university-operated vivarium with a standard 12-hour light-dark cycle. Animals were weighed every 3 days to ensure appropriate growth and health post injury. Animals were housed in the vivarium at a minimum of 7 days to allow for stabilization of gut microbiomes prior to initiating experiments.

\section{Experimental Groups}

Experiments were conducted in multiple cohorts at two different time points based on the treatment administered. Experiments lasted for either 72 hours as an acute post-injury model or 21 days as a 
chronic post-injury model. Within both time points, all injured animals either received daily probiotics, antibiotics, or no treatment. Sham-injured animals were included in the experiments to serve as controls.

\section{Controlled Cortical Impact (CCl)}

Animals were anesthetized with $4 \%$ isoflurane and oxygen in a vented chamber and then maintained at 2$3 \%$ isoflurane for the duration of the procedure. All animals underwent a venous blood draw via tail vein prior to the injury. The animal was then secured on a stereotactic frame and the surgical site was prepped with alcohol and iodine solution. Subcutaneous $0.25 \%$ bupivacaine was administered prior to incision for local anesthesia. A midline cranial incision was made, and the right sided musculature and soft tissue was bluntly dissected away for exposure of the calvarium. A 7-mm diameter craniectomy was performed between the right coronal and lambdoid sutures. A controlled cortical impact device (Impact One Stereotaxic Impactor, Leica Microsystems, Buffalo Grove, IL) was utilized to administer a standardized and unilateral severe brain injury as previously described (18). Severe injury parameters included a depth of $3.1 \mathrm{~mm}$, impact velocity of $5.6 \mathrm{~m} / \mathrm{s}$, and a dwell time of $200 \mathrm{~ms}$ using a $6 \mathrm{~mm}$ diameter impactor tip. This allowed for a severe injury to the parietal association cortex. Immediately after the injury, the incision was closed using sterile wound clips and animals were allowed to recover in newly cleaned micro-isolator cages provided by the University Vivarium. Sham injured animals underwent the same procedures outlined above but did not receive a craniectomy or severe injury via the impactor tip. Animals were observed post-operatively and monitored on a daily basis for pain and wound complications in compliance with ARRIVE guidelines.

\section{Probiotic Preparation and Administration}

Human breast milk derived Lactobacillus reuteri DSM 17938 (LR 17938) was provided by BioGaia AB (Stockholm, Sweden) and is commercially available. LR 17938 was cultured in deMan-Rogosa-Sharpe (MRS) medium, anaerobically, at $37^{\circ} \mathrm{C}$ for 16 hours. Quantitative analysis of bacteria in culture media was performed by comparing the absorbance at $600 \mathrm{~nm}$ to cultures of known concentrations using a standard curve of colony forming units (CFUs) per milliliter $(\mathrm{mL})$ using an Eppendorf Photometer. LR 17938 was inoculated in fresh media every 2-3 days to ensure viability and to prevent contamination. Animals were given a probiotic dose of $10^{8} \mathrm{CFU} /$ day, daily, by orally gavage, starting on the day of injury until the day of sacrifice.

\section{Antibiotic Preparation and Administration}

Broad spectrum antibiotics were utilized to instigate gut dysbiosis. Animals were treated with $50 \mathrm{mg} / \mathrm{kg} / \mathrm{day}$ of Ampicillin (GoldBio), $50 \mathrm{mg} / \mathrm{kg} /$ day Gentamicin (GoldBio), and $50 \mathrm{mg} / \mathrm{kg} / \mathrm{day}$ Metronidazole (Sigma) in their drinking water and allowed to drink ad libitum. Antibiotic treatment was started 7 days prior to injury and continued for either 72 hours or 21 days depending on the treatment group the animal was assigned to.

\section{Microglia Isolation and Characterization}


Animals were euthanized at either 72 hours or 21 days after injury. Brains were harvested and processed as previously described by our lab (19) utilizing the Neural Tissue Dissociation Kit and GentleMACS dissociator (Miltenyi Biotec). Myelin was removed by Percoll centrifugation. CD11+ enrichment was performed with CD11b/c microbeads and quadraMACS magnet (Miltenyi Biotec). Microglia antibody staining panels were based on M1 and M2 gating strategies (5).

The microglia were immunophenotyped using multiparametric flow cytometry panel. Data were acquired on LSR-2 flow cytometer (BD Biosciences). Using a modification of previously published methods, identification of microglia was achieved with a triplet anchor gating strategy that isolated CD45+, $\mathrm{CD} 11 \mathrm{bc}+$, and P2Y12+ cells (5). Cells that were CD45+, CD11bc+, but P2Y12 negative were considered infiltrating myeloid/macrophage populations (Supplementary Figure 1)(5). Pro-inflammatory (M1) markers (CD32+ and CD86+) and anti-inflammatory (M2) markers (CD200R+, RT1B+, and CD163+) were identified under this microglia gating strategy. Absolute microglia cell count was determined by calculating the number of cells per milligram of tissue. Brain hemispheres were weighed prior to cell isolation and incorporated into the dilution factors to achieve this number. To analyze these data, traditional analysis of comparing absolute number or percentage or mean fluorescence intensity of the microglia with different treatments were performed.

\section{Cell Isolation and Immune Characterization of Gut-Associated Lymphoid Tissue}

Animals were euthanized at either 72 hours or 21 days after injury. Upon sacrifice, Peyer's patches (PP) and mesenteric lymph nodes (MLN), also known as gut-associated lymphoid tissue (GALT), were harvested for cell isolation. MLNs underwent mild tissue dissociation with collagenase and DNAse and incubated for 45 minutes. PPs underwent mild tissue dissociation with incubation in spleen dissociation media (STEMCELL) for 45 minutes. Both tissue samples were then filtered through 70-micron cell strainers prior to cell staining. Samples were stained for general myeloid and lymphoid makers. The myeloid antibody panel consists of the following markers: HIS48-FITC, CD43-PE, CD8a-PerCP, CD11b/cPECy7, CD4-APCCy7, CD172a-V450. The lymphoid antibody panel consists of the following markers: CD3-FITC, CD25-PE, CD8a-PerCP, CD11b/c-PECy7, RT1B-APC, CD4-APCCy7, CD45RA-V450. Data for the GALT samples were acquired on a Galios Flow Cytometer (Beckman Coulter). Subsequent data analyses were completed utilizing Kaluza software (Beckman Coulter).

\section{Statistical Analyses}

Flow cytometry data were processed and analyzed using Kaluza software (Beckman Coulter) and FlowJo software (FlowJo, LLC). Data were analyzed using GraphPad Prism (GraphPad Software, Inc., La Jolla, CA). Analysis was focused on the injured cerebral hemisphere (ipsilateral). Comparisons between means of each group were analyzed using one-way ANOVA with Dunnett's post-hoc test. Comparisons between ratios were analyzed using a Student's T-test. ROUT analyses (1\%) identified outliers in the data, which were excluded in the final analysis. All group data are presented as mean \pm standard error. Values of $p \leq$ 0.05 were considered significant. Statistical significance is indicated with $(*)$ for $p \leq 0.05$, (**) for $p \leq$ $0.01,(* * *)$ for $p \leq 0.001$, and $(* \star * *)$ for $p \leq 0.0001$. 


\section{Bacterial 16S rRNA Gene Sequencing and Analyses}

All animals were housed individually to prevent cross-colonization of rats in the same cage. Fecal pellets were collected at baseline prior to the start of each experiment, and then collected every 3 days post injury. Fecal pellets were collected utilizing clean catch procedures in an open field and stored in sterile tubes, which were then immediately frozen and stored at $-80^{\circ} \mathrm{C}$.

Sample preparation and analysis was conducted by the Alkek Center for Metagenomics and Microbiome Research (CMMR) at Baylor College of Medicine. In brief, bacterial genomic DNA was extracted using the Qiagen MagAttract PowerSoil DNA Kit (formerly sold by MO BIO as PowerMag Soil DNA Isolation Kit). The 16S rDNA V4 region was amplified by PCR and sequenced in the MiSeq platform (Illumina) using the $2 \times 250$ bp paired-end protocol yielding pair-end reads that overlap almost completely. The primers used for amplification contain adapters for MiSeq sequencing and single-index barcodes so that the PCR products may be pooled and sequenced directly (20), targeting at least 10,000 reads per sample. $16 \mathrm{~Sv} 4$ rDNA sequences are clustered into Operational Taxonomic Units (OTUs) at a similarity cutoff value of $97 \%$ using the UPARSE algorithm (21). OTUs are subsequently mapped to an optimized version of the SILVA Database (22) containing only sequences from the v4 region of the 16S rRNA gene to determine taxonomies. Abundances are recovered by mapping the demultiplexed reads to the UPARSE OTUs. Downstream analyses and statistical analyses were utilized using a visual toolkit developed at CMMR named ATIMA (Agile Toolkit for Incisive Microbial Analyses). ATIMA allows for identifying trends in taxa abundance, alpha diversity (richness and evenness), beta diversity (in-between sample differences), Principal Coordinate Analysis (PCoA) ordinations, and heatmaps. Statistical significance was determined using non-parametric Mann-Whitney tests for two category comparisons or the Kruskal-Wallis test when comparing three or more categories. PCoA plots utilize Monte Carlo permutation tests to estimate pvalues.

\section{Study Approval}

All protocols involving the use of animals were approved by the University of Texas Health Science Center at Houston Animal Care and Use Committee (AWC 18-0011). Protocols involved were in compliance with the National Institutes of Health Guide for the Care and Use of Laboratory Animals.

\section{Data Availability}

The data that support findings of this study are available from the corresponding author upon request.

\section{Results}

Seventy-two hours after $\mathrm{CCl}$, probiotic and antibiotic treatment do not affect pro or anti-inflammatory surface markers. 
Seventy-two hours after $\mathrm{CCl}$, there was an increase in the number of microglia when compared to Sham [Fig. 1A, Sham: $n=7,156394 \pm 54430$ vs CCl: $n=8,3622788 \pm 2021391(p=0.41)]$. There was an increase in microglia in CCl-Abx vs. CCI [CCl-Abx: $n=11,5760252 \pm 2268494(p=0.68)]$. There was no differences in the number of microglia when comparing CCI-Pro vs CCl animals [CCI-Pro: $n=11,3109550 \pm 765960$ ( $p$ $=0.99$ ). In addition, we examined the phenotype of microglia after injury and treatment. There were no significant differences of either probiotics or antibiotics when comparing pro-inflammatory surface markers (CD 32 and CD86) to CCl alone (Figs. 1BC). Anti-inflammatory surface markers (CD200r, RT1B and CD163) were also not significant when compared to $\mathrm{CCl}$ alone (Figs. DEF).

Twenty-one days after $\mathrm{CCl}$, probiotic and antibiotic treatment increase anti-inflammatory surface markers.

Twenty-one days after $\mathrm{CCl}$, there were no significant differences in absolute microglia between the groups (Fig. 2A). There were no differences in pro-inflammatory surface markers in either treatment when compared to $\mathrm{CCl}$ alone (Figs. 2BC). However, after injury and probiotic treatment (CCI-Pro), there was a significant increase in anti-inflammatory surface marker CD200r when compared to $\mathrm{CCl}$ alone [Fig. 2D, CCI: $n=14,7.1 \% \pm 1.7 \%$ vs CCI-pro: $n=16,5.4 \% \pm 1.2 \%(p=0.0001)]$. There were no significant differences of either probiotics or antibiotics when comparing pro-inflammatory surface markers (CD32 and CD86) to $\mathrm{CCl}$ alone (Figs. 2BC). Interestingly, surface marker CD163 was significantly greater with antibiotic treatment (CCl-Abx) vs $\mathrm{CCl}$ alone (Fig. 2F). There were no differences between $\mathrm{CCl}$ and treatments with RT1B (Fig. 2E). RT1B. There were significant increases in the CCl vs sham for CD86 [Fig. 2C, Sham:n $=14$, $23.24 \% \pm 2.2 \%$ vs CCl: $n=14,41.48 \% \pm 6.1 \%(p=0.0046)]$ and Rt1b [Fig. $2 E$, Sham: $n=14,23.49 \% \pm 1.4 \%$ vs CCl:n $=14,45.61 \% \pm 4.5 \%(p=0.0005)]$.

\section{Twenty-one days after $\mathrm{CCl}$, probiotic treatment attenuates $\mathrm{M} 1: \mathrm{M} 2$ ratio.}

Seventy-two hours after treatments, there were no significant differences in M1:M2 (Fig. 2AB). However, 21 days after treatment, probiotic treatment resulted in a decrease in $\mathrm{M} 1: \mathrm{M} 2$ when compared to $\mathrm{CCl}$ alone of surface markers CD86: CD200r $(p=0.0007$, Fig. $3 C)$. Probiotic treatment also significantly decreased CD32:CD200r ( $p=0.0214$, Fig. $3 C$ ) when compared to $\mathrm{CCl}$ alone. There were no significant differences in the other surface marker comparisons (Fig. 3CD).

\section{Seventy-two hours after $\mathrm{CCl}$, antibiotic treatment increases natural killer cells in Peyer's Patches.}

Antibiotic treatment with injury results in a significant increase in Natural Killer cells (Cd161+) isolated from PP when compared to $\mathrm{CCl}$ alone 72 hours after injury [Fig. $4 \mathrm{~A}, \mathrm{CCl}: \mathrm{n}=8,0.34 \pm 0.12$ vs $\mathrm{CCl}-\mathrm{Abx}: \mathrm{n}=$ $12,0.88 \pm 0.30(p<0.0001)]$. Neither antibiotic or probiotic treatment affected the monocytes derived from PP, however there was an overall significance as measured by One-way ANOVA ( $p<0.05$, Fig. 4B).

Probiotic treatment did result in a decrease in the percentage of monocytes in PP when compared to $\mathrm{CCl}$ alone (Fig. 4B). Additionally, there were no differences in the CD11 bc+, T-regulatory cells and CD3 + cells at 72 hours between probiotics, antibiotics, $\mathrm{CCl}$ and Sham. 
Twenty-one days after TBI, antibiotic and probiotic treatments increases monocytes, CD11bc+, natural killer cells and CD3 + cells in Peyer's Patches.

Peyer's patches (PP) are an integral part of the gut's immune response and serves as part of the gutassociated lymphoid tissue (GALT).

MONOCYTES: With antibiotic treatment (CCl-Abx: $n=6,26 \pm 2.1)$, there was a significant increase $(\mathrm{p}<$ $0.0001)$ in monocytes (CD172a+) when compared to $\mathrm{CCl}(\mathrm{CCl}: \mathrm{n}=4,1.5 \pm 0.84$, Fig. 5A). Additionally, probiotic treatment (CCI-Pro: $n=8,6.2 \pm 2.6)$ also resulted in a significant increase $(p=0.0034)$ in comparison to $\mathrm{CCl}$ (Fig. 5A). There were no differences between sham and $\mathrm{CCl}$.

CD11bc+: Antibiotic treatment (CCl-Abx: $n=8,13.5 \pm 2.1)$ resulted in a significant increase $(p<0.002)$ in $\mathrm{CD} 11 \mathrm{bc}+$ cells when compared to $\mathrm{CCl}(\mathrm{CCl}: \mathrm{n}=4,7.4 \pm 4.7$, Fig. 5B). Surprisingly, probiotic treatment (CClPro: $n=8,3.8 \pm 1.1$ ) was not significant in comparison to $\mathrm{CCl}$ (Fig. $5 \mathrm{~B})$. There were no differences between sham and $\mathrm{CCl}$.

Natural Killer cells (CD161+): Antibiotic treatment (CCI-Abx:n $=8,1.4 \pm 0.5)$, resulted in a significant increase $(p<0.001)$ in CD161 + cells when compared to $\mathrm{CCl}(\mathrm{CCl}: n=7,0.3 \pm 0.4$, Fig. $5 \mathrm{C})$. Probiotic treatment (CCI-Pro: $\mathrm{n}=8,0.8 \pm 0.4$ ) was not significant in comparison to $\mathrm{CCl}$ (Fig. $5 \mathrm{C}$ ). There were no differences between sham and $\mathrm{CCl}$.

T Regulatory Cells (CD3 + CD 4 + CD25+): Antibiotic treatment nor Probiotic treatment affected T Regulatory Cells (graph not shown).

CD3+: Probiotic treatment (CCI-Pro: $n=7,45 \pm 9)$, resulted in a significant increase $(p<0.05)$ in CD3 + cells when compared to $\mathrm{CCl}(\mathrm{CCl}: \mathrm{n}=10,23 \pm 19$, Fig. 5D). Antibiotic treatment (CCl-Abx: $n=8,30 \pm 10)$ was not significant in comparison to $\mathrm{CCl}$ (Fig. 5D). There were no differences between sham and $\mathrm{CCl}$.

\section{Antibiotics induce a pro-inflammatory immune response in the Mesenteric Lymph Nodes after 21 days post-injury but not at 72 hours post-injury.}

The mesenteric lymph nodes (MLN) are a component of the gut-associated lymph tissue (GALT), and act as mediator between the intestinal tract and the rest of the body. At 72 hours, there were no clear relationships seen among the lymphoid and T cells makers (data not shown). However, 21 days postinjury, there was an increase in CD11bc+ $(p<0.01)$ due to Abx treatment (CCI-Abx: $n=6,0.79 \pm 0.16)$ vs $\mathrm{CCl}(\mathrm{CCl}: n=5,0.26 \pm 0.06)$. In addition, there was an increase $(p<0.05)$ in and CD $161+$ cells in CCl-Abx (CCl-Abx: $n=7,0.85 \pm 0.22)$ in comparison to $\mathrm{CCl}$ alone $(\mathrm{CCl}: \mathrm{n}=6,0.27 \pm 0.08)$. There were no differences in $\mathrm{CCl}-\mathrm{Pro}$ vs $\mathrm{CCl}$.

Fecal Microbiome Analysis: time-course between injuries and treatment with probiotics at baseline versus post-injury day 3 
Fecal analyses were performed in order to study the effects our experimental design on the animals' microbiome. Overall, there were no significant changes to the fecal microbiome after a severe $\mathrm{CCl}$ injury and there were no significant changes to the fecal microbiome with treatment of probiotic, Lactobacillus reuteri (LR). The $₫$ diversity can be portrayed as Observed Organized Taxonomic Units (OTUs) and as

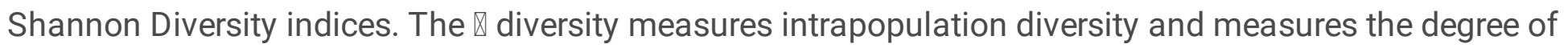
richness and/or evenness within each sample. Baseline samples were collected prior to the start of each experiment, and day 3 sample was collected on post-CCI injury or post-sham injury day 3 . Animals that did not receive any type of treatment are representative of a control cohort for our microbiome analyses. There were no microbiome changes seen within the sham animals over 3 days (Figs. 7ABCD). The data also demonstrates the $\otimes$ diversity for Sham and $\mathrm{CCl}$ animals that received probiotics for 3 days after sham or $\mathrm{CCl}$ injury respectively. $\mathrm{CCl}$ alone did not cause a significant change in the $₫$ diversity over a time course of 3 days. The $\beta$ diversity represents the interpopulation diversity allowing for cluster visualization of samples (Figs. 7EFGH). There were no significant changes in beta diversity over a time course of 3 days with and without treatment of probiotics.

\section{Fecal Microbiome Analysis: time-course between injuries and treatment with antibiotics at baseline versus post-injury day 3}

Fecal microbiome analyses were also performed on animals that received treatment with broad-spectrum antibiotics in order to confirm induced gut-dysbiosis. The use of broad-spectrum antibiotics caused a significant gut-dysbiosis in both Sham and $\mathrm{CCl}$. There was significant reduction of the $\nabla$ diversity within experimental groups receiving antibiotic treatment (Figs. 8ABCD). Baseline fecal samples were collected prior to antibiotic treatment and injury. The day 3 fecal samples were collected on post-injury day 3 . Antibiotic treatment also caused a significant shift in the $\beta$ diversity within Sham and $\mathrm{CCl}$ animals (Figs. 8EF). We found that samples in both experimental groups clearly clustered together and shifted significantly after treatment with antibiotics, confirming an induced gut dysbiosis in these animals (Figs. 8EF). Analysis of relative abundance based on most abundant phyla isolated from fecal samples before and after treatment with antibiotics revealed that the entire experimental cohort that received antibiotics clearly demonstrated dramatic changes within the most abundant phyla (Figs. 8GH). Further stratification between Sham and $\mathrm{CCl}$ animals indicated that there are more dramatic changes in the relative abundance within the $\mathrm{CCl}$ animals when compared to the Sham animals. Statistical differences between the two experimental groups that were treated with antibiotics revealed that antibiotics cause a significant dysbiosis in sham animals. Furthermore, the dysbiosis seen is significantly worsened in setting of severe $\mathrm{CCl}$ injury (Figs. 8I).

\section{Discussion}

Traumatic brain injury is a systemic injury that effects the gastrointestinal tract (GI). Disruption in the GI due to TBI results in a systemic inflammation which is dependent upon the gut microbiome (GM) (8). Our results demonstrate that there is a direct relationship between gut dysbiosis and amplification of the microglial inflammatory response after severe traumatic brain injury. 
The interaction between the gut and the brain is complex and mediated through an intricate system of immune cells and cytokines. Any factors that cause a shift in the microbiome secondary to trauma or physiological stress is likely to play a role in disease exacerbation and functional recovery, especially within the pathophysiology of traumatic brain injury (TBI) (23). Contrary to other reports, the 16S rRNA sequencing analysis did not demonstrate changes in the microbiome after an isolated CCl injury (24). Treatment with probiotic, Lactobacillus reuteri DSM 17938 (LR 17938), did not produce any significant changes in the fecal microbiome profiles in either Sham or CCI (Fig. 7). However, antibiotic-induced dysbiosis resulted in an amplified cellular immune response within the GALT (Figs. 4,5,6,8). Furthermore, while probiotics did not shift the microbiome profile (Fig. 7), a dampened pro-inflammatory immune response and attenuation of microglial pro-inflammatory markers was demonstrated (Fig. 3D and 2F). In several models of autoimmune and inflammatory diseases of the gut, the action of probiotics is thought to be mediated between the mesenteric lymph nodes and the systemic immune system (25). L. reutieri can also affect T-regulatory cells within the small bowel mucosa after only a few days of administration (26). These communications may not entirely be reflected downstream through the colon and rectum microbiome profiles measured by stool.

Our study aimed to characterize the immune response in the gut associated lymphoid tissue (GALT) to better understand the cross communication between the gut and brain in the setting of injury and manipulation of the microbiome. The GALT is comprised of the Peyer's patches (PP) and the mesenteric lymph nodes (MLN). The PPs are lymphoid follicles scattered throughout the intestinal tract and serve to mount a protective immune response while continuously surveying the gut-microbe environment. From the PPs, cellular signals drain into the MLNs, which then mount a systemic response in order to maintain homeostasis during physiological stress (16). We sought to isolate cells from the GALT and utilize myeloid and lymphoid immune cell markers to better understand the function the gut plays in the postinjury immune response. Our data indicate that there is an overall upregulated myeloid response in both the PPs and MLNs after TBI with antibiotic-induced gut dysbiosis (Figs. 5 and 6). There is an intimate relationship between the peripheral myeloid response and the central immune response after TBI. With disruption of the blood brain barrier after a severe TBI, peripheral immune cells are able to infiltrate the brain and exert an amplified inflammatory effect on the site of injury $(27,28)$. Therefore, if antibioticinduced gut dysbiosis is driving the peripheral immune response to injury, its effects may also infiltrate the blood brain barrier after a severe TBI and contribute to microglia M1 activation. In contrast, the same may be extrapolated from use of probiotics. If probiotics exert a systemic cellular anti-inflammatory response after injury, there is potential to cross the blood brain barrier after a severe TBI and contribute to microglia M2 activation.

Microglia play a key role in the pathophysiology in TBI, in that the dysregulated activation propagates an inflammatory environment that becomes destructive and detrimental leading to long-term disabilities (6). As previously described, a severe $\mathrm{CCl}$ injury results in an increase in activated microglia, as well as a shift towards the pro-inflammatory M1 phenotype, which is associated with destructive and cytotoxic activity to surrounding tissues $(2,29)$. We found this to be true at two different timepoints: 72 hours (acute) and 21 days (chronic). This confirms previous reports of M1 polarization of microglia after a severe injury, 
and showed that there is sustained activation and dysregulation of microglia up to 21 days after injury (6). Kigerl et al. demonstrated that gut dysbiosis impairs recovery after spinal cord injury (30). They manipulated the gut via using antibiotics and probiotics to demonstrate the unique connection between the gut and the central nervous system. They concluded that by reversing gut dysbiosis, outcomes were improved in mice that underwent spinal cord injury. Similarly, our aim was to manipulate the gut microbiome using a single strain probiotic or a mixture of broad-spectrum antibiotics in order to visualize an effect on microglia activation after experimental $\mathrm{CCl}$ in rats. Probiotic treatment ( 21 days) after severe $\mathrm{CCl}$ injury helped decrease the microglia M1:M2 ratio (Fig. 3C). Specifically, CD200R increased with probiotic treatment after injury at 21 days (Fig. 2D). CD200R-dependent inhibitory signaling prevents bacterial infections and attenuates neuroinflammation in rodent stroke models (31). In contrast, animals that received antibiotics prior to injury in order to induce a gut-dysbiosis, we demonstrated a significant shift towards a pro-inflammatory state consistent with an M1 phenotype.

Our data indicate after a severe $\mathrm{CCl}$ injury, probiotics have an overall anti-inflammatory effect on microglia. We found an overall attenuation of microglial activation after 72 hours post-injury and treatment with probiotics. Conversely, animals that received antibiotics prior to injury in order to induce a gut dysbiosis, had a significantly elevated number of activated microglia 72 hours after injury. Similar results were seen at 21 days post injury. A key difference is a decreased effect of the dysbiosis on microglia activation and a continued activation with the use of probiotics. While at 21 days we found an increase in activated microglia in our probiotic treated animals, this ultimately did not change the M1/M2 profile trends seen at 72 hours. Even though there are more microglia active at 21 days post injury with probiotics, these microglia favored an anti-inflammatory M2 phenotype, which correlates with a pro-repair and scavenging state rather than a cytotoxic state.

Our data demonstrate that the anti-inflammatory effect of probiotic LR 17938 is mediated through a T-cell mediated response, more so in the PPs than the MLNs (Fig. 5), and especially with long-term use. The Tcell response after injury is blunted, especially with the treatment of antibiotics. This suggests that an attenuated T-cell response after injury correlates with an amplified pro-inflammatory response. The therapeutic effects of LR 17938 (LR) may be due to a dampening effect of the T-cell response to injury. He et al. demonstrated that LR was able to remodel the gut microbiome in an autoimmune encephalomyelitis rodent model by acting on T-regulatory cell dysfunction (32). Studies have shown that use of probiotics, especially those containing lactobacillus, upregulate $T$ regulatory cell activity, reducing inflammation and reverting back toward homeostasis (33). Therefore, resetting a dysbiotic gut after a traumatic injury with the use of probiotics may have potential as a therapeutic target.

Studying the effects of gut dysbiosis provides valuable insight on the mechanism of action between the gut and the brain. Efferent pro-inflammatory signaling after TBI induces ileus and increases gut mucosal paracellular permeability, amplifying systemic inflammatory responses (34). It has been previously shown that dysbiosis induces a pro-inflammatory reaction and exacerbates lesion volume and functional deficits in response to ischemic stroke in a rodent model (9). Depletion of gut microbiota with the use of broad-spectrum antibiotics was found to also worsen outcomes in a rodent stoke model (35). Further, 
Kigerl et al. demonstrated worsening post-injury lesion volume and an increased inflammatory response after antibiotic induced dysbiosis (30). Our data confirms these findings in that antibiotic-induced gut dysbiosis amplified the post-injury immune response. The $16 \mathrm{~s}$ rRNA fecal bacterial sequencing results further highlights this pathophysiology. Manichanh et. al studied the effect of gut dysbiosis in a rodent model where they altered the gut-microbiome with a fecal transplant and antibiotic treatment. Their results showed that rats that underwent a fecal transplant without antibiotic treatment were more resistant to microbiome changes, whereas their microbiome exhibited more plasticity when pretreated with antibiotics, suggesting that an altered microbiome significantly alters homeostatic pathways (36). When comparing Sham and CCI injury animals treated with antibiotics, we found that there was significantly more gut dysbiosis in the setting of injury, which can be linked to amplification of proinflammatory markers in both the microglia and the GALT.

There are several limitations to our study. Our data exists through a rodent model, which can be challenging to correlate clinically in humans. However, this study aims to understand how clinically relevant manipulations of the gut can affect the inflammatory response. There are an increasing number of studies investigating the connection between the gut microbiome and the immune response to injury. Potential alternative experimental designs to address our observed phenomena include the use of germfree animals with and without dysbiotic fecal transplants. These models have their own limitations as well and are less translationally relevant. Further, our experiments did not aim to conduct functional rodent studies, which focuses on assessing spatial learning and memory after neurological injury through behavioral tests such as the Morris Water Maze (37). This data would demonstrate the therapeutic endpoint in targeting the neuroinflammatory response following brain injury, however, it is reasonably established in the literature that chronic neuroinflammation, more specifically chronic microglial activation, is correlated with clinical neurodegeneration and neurocognitive impairment in both rodent and human models $(38,39)$. Ramlackhansignh et al. studied patients with head injury over an extended period of time with the use of positron emission tomography. They demonstrated the significant effect that chronic activation of microglia has on the structural integrity of the human brain (23). These established findings suggest that focusing therapeutic strategies in attenuating microglial activation and overall systemic inflammation after TBI will allow for improved clinical outcomes in this patient population (40).

Clinical studies are demonstrating similar data to laboratory models. Nicholson et al. conducted a prospective study in severely injured trauma patients and demonstrated gut dysbiosis in trauma patients when compared to healthy controls (41). They also demonstrated that the level of microbiome changes can be associated with injury severity and transfusion of blood products (42). Howard et al. conducted another prospective clinical study in trauma patients that also reported a significant changes in microbiome profiles within 72 hours of severe traumatic injury (43). There is growing evidence that alterations in microbiome phenotypes can be correlated with clinical outcomes. While associations can be extrapolated from the data, our findings do not necessarily prove causality. Multiple studies have already shown the effectiveness in early post-traumatic enteral feeding on overall patient outcomes, and is currently supported in the guidelines by the Society of Critical Care Medicine $(12,13,44)$. However, 
there is no general consensus among the medical community on the use of probiotics in critically ill patients or trauma patients. Probiotics are considered a food supplement by the Food and Drug Administration (FDA), and are regulated in the same category as vaccines. Therefore, the investigation of probiotics will need to be conducted within a regulatory environment due to safety concerns and lack of standardization and evidence-based medicine comparing varying strains, dosages, timing of therapy (14). While further investigation is needed to elucidate the mechanism of action of how probiotics act in order to exert an anti-inflammatory effect in both pre-clinical and clinical models, it can be concluded from our results that targeting a dysfunctional gut may help the body regain homeostasis through antiinflammatory signaling.

\section{Abbreviations}

Traumatic brain injury (TBI)

Cortical Contusion Injury (CCl)

Gastrointestinal Tract (GI)

Gut Microbiome (GM)

Gut-Associated Lymphoid Tissue (GALT)

Central Nervous System (CNS)

Peyer's Patches (PP)

Mesenteric Lymph Nodes (MLN)

Analysis of Variance (ANOVA)

\section{Declarations}

\section{Acknowledgements}

The authors thank UT Health Animal Welfare Committee and the Center for Laboratory Animal Medical and Care for their support and care of our animals. We thank Dr. Melissa Mezzari and the Alkek Center for Metagenomics and Microbiome Research (CMMR) at Baylor College of Medicine for processing our fecal samples and guiding our microbiome analysis. We thank Dr. J. Marc Rhoads and Dr. Yuying Liu from the UT Department of Pediatric Gastroenterology for helping with designing preliminary experiments and assisted to anaerobically culture, and quantify the probiotic stain used in this study. We acknowledge that BioGaia AB (Stockholm, Sweden) owns property rights of the probiotic strain, Lactobacillus reuteri DSM 17938 , and has allowed our lab to use this strain for research purposes only.

\section{Conflict of Interest Statement}


Dr. Cox has Scientific Advisory Board positions with CBR, Inc., Biostage, Inc., and Cellvation, Inc.; Equity/royalty interest in EMIT, Corp., Coagulex, Inc., Cellvation, Inc., and Biostage, Inc.

1. Ethics approval and consent to participate: Yes

2. Consent for publication: Yes

3. Availability of data and material: Yes

4. Competing interests: Dr. Cox has Scientific Advisory Board positions with CBR, Inc., Biostage, Inc., and Cellvation, Inc.; Equity/royalty interest in EMIT, Corp., Coagulex, Inc., Cellvation, Inc., and Biostage, Inc.

5. Funding: Glassell Family Foundation and the Pediatric Surgery Research Fund at the University Texas Health Science Center in Houston.

6. Author Contributions:

Akshita Kumar, MD, MS: Design, execution, analysis and writing

Supinder S. Bedi Ph.D.: Analysis and writing

Naama Toledano-Furman, Ph.D. : Analysis

Louis Carrillo, MD, MPH: Execution

Fanni Cardenas, MD, MPH: Execution

Henry W. Caplan, MD: Execution

David Sequeira: Execution

Allison L. Speer, MD: Analysis

Scott D. Olson, Ph.D. : Analysis

Charles E Wade, Ph.D. : Analysis

Charles S. Cox Jr., MD: Design and writing

7. All protocols involving the use of animals were in compliance with the National Institutes of Health Guide for the Care and Use of Laboratory Animals and were approved by the University of Texas Health Science Center Institutional Animal Care and Use Committee.

\section{References}

1. Graeber MB. Changing face of microglia. Science. 2010;330(6005):783-8. 
2. Bedi SS, Hetz R, Thomas C, Smith P, Olsen AB, Williams S, et al. Intravenous multipotent adult progenitor cell therapy attenuates activated microglial/macrophage response and improves spatial learning after traumatic brain injury. Stem Cells Transl Med. 2013;2(12):953-60.

3. Csuka E, Hans VH, Ammann E, Trentz O, Kossmann T, Morganti-Kossmann MC. Cell activation and inflammatory response following traumatic axonal injury in the rat. Neuroreport. 2000;11(11):258790.

4. Davalos D, Grutzendler J, Yang G, Kim JV, Zuo Y, Jung S, et al. ATP mediates rapid microglial response to local brain injury in vivo. Nat Neurosci. 2005;8(6):752-8.

5. Toledano Furman NE, Prabhakara KS, Bedi S, Cox CS Jr, Olson SD. OMIP-041: Optimized multicolor immunofluorescence panel rat microglial staining protocol. Cytometry A. 2018;93(2):182-5.

6. Loane DJ, Kumar A. Microglia in the TBI brain: The good, the bad, and the dysregulated. Exp Neurol. 2016;275 Pt 3:316 - 27.

7. Madathil SK, Wilfred BS, Urankar SE, Yang W, Leung LY, Gilsdorf JS, et al. Early Microglial Activation Following Closed-Head Concussive Injury Is Dominated by Pro-Inflammatory M-1 Type. Front Neurol. 2018;9:964.

8. Bansal V, Costantini T, Kroll L, Peterson C, Loomis W, Eliceiri B, et al. Traumatic brain injury and intestinal dysfunction: uncovering the neuro-enteric axis. J Neurotrauma. 2009;26(8):1353-9.

9. Singh V, Roth S, Llovera G, Sadler R, Garzetti D, Stecher B, et al. Microbiota Dysbiosis Controls the Neuroinflammatory Response after Stroke. The Journal of Neuroscience: the official journal of the Society for Neuroscience. 2016;36(28):7428-40.

10. Kharrazian D. Traumatic Brain Injury and the Effect on the Brain-Gut Axis. Altern Ther Health Med. 2015;21(Suppl 3):28-32.

11. Olsen $A B$, Hetz RA, Xue H, Aroom KR, Bhattarai D, Johnson E, et al. Effects of traumatic brain injury on intestinal contractility. Neurogastroenterol Motil. 2013;25(7):593-e463.

12. Azim A, Haider AA, Rhee P, Verma K, Windell E, Jokar TO, et al. Early feeds not force feeds: Enteral nutrition in traumatic brain injury. J Trauma Acute Care Surg. 2016;81(3):520-4.

13. Chiang YH, Chao DP, Chu SF, Lin HW, Huang SY, Yeh YS, et al. Early enteral nutrition and clinical outcomes of severe traumatic brain injury patients in acute stage: a multi-center cohort study. $J$ Neurotrauma. 2012;29(1):75-80.

14. Urben LM, Wiedmar J, Boettcher E, Cavallazzi R, Martindale RG, McClave SA. Bugs or drugs: are probiotics safe for use in the critically ill? Curr Gastroenterol Rep. 2014;16(7):388.

15. Kim H-J, Lee J-H, Kim S-H. Therapeutic effects of human mesenchymal stem cells on traumatic brain injury in rats: secretion of neurotrophic factors and inhibition of apoptosis. $J$ Neurotrauma. 2010;27(1):131-8.

16. Blumberg RS. Inflammation in the intestinal tract: pathogenesis and treatment. Dig Dis. 2009;27(4):455-64.

17. Elmore SA. Enhanced histopathology of the lymph nodes. Toxicol Pathol. 2006;34(5):634-47. 
18. Lighthall JW. Controlled cortical impact: a new experimental brain injury model. J Neurotrauma. 1988;5(1):1-15.

19. Toledano Furman NE, Prabhakara KS, Bedi S, Cox CS, Olson SD. Rat Microglia Isolation and Characterization Using Multiparametric Panel for Flow Cytometric Analysis. In: Srivastava AK, Cox CS, editors. Pre-Clinical and Clinical Methods in Brain Trauma Research. New York: Springer New York; 2018. pp. 191-9.

20. Caporaso JG, Lauber CL, Walters WA, Berg-Lyons D, Huntley J, Fierer N, et al. Ultra-high-throughput microbial community analysis on the Illumina HiSeq and MiSeq platforms. ISME J. 2012;6(8):16214.

21. Edgar RC. UPARSE: highly accurate OTU sequences from microbial amplicon reads. Nat Methods. 2013;10(10):996-8.

22. Quast C, Pruesse E, Yilmaz P, Gerken J, Schweer T, Yarza P, et al. The SILVA ribosomal RNA gene database project: improved data processing and web-based tools. Nucleic Acids Res. 2013;41(Database issue):D590-6.

23. Ramlackhansingh AF, Brooks DJ, Greenwood RJ, Bose SK, Turkheimer FE, Kinnunen KM, et al. Inflammation after trauma: microglial activation and traumatic brain injury. Ann Neurol. 2011;70(3):374-83.

24. Nicholson SE, Watts LT, Burmeister DM, Merrill D, Scroggins S, Zou Y, et al. Moderate Traumatic Brain Injury Alters the Gastrointestinal Microbiome in a Time-Dependent Manner. Shock (Augusta, Ga). 2018.

25. Liu Y, Alookaran JJ, Rhoads JM. Probiotics in Autoimmune and Inflammatory Disorders. Nutrients. 2018;10(10).

26. Liu Y, Fatheree NY, Dingle BM, Tran DQ, Rhoads JM. Lactobacillus reuteri DSM 17938 changes the frequency of Foxp3 + regulatory $T$ cells in the intestine and mesenteric lymph node in experimental necrotizing enterocolitis. PLoS One. 2013;8(2):e56547.

27. Clark RS, Schiding JK, Kaczorowski SL, Marion DW, Kochanek PM. Neutrophil accumulation after traumatic brain injury in rats: comparison of weight drop and controlled cortical impact models. $J$ Neurotrauma. 1994;11(5):499-506.

28. Harting MT, Jimenez F, Adams SD, Mercer DW, Cox CS. Jr. Acute, regional inflammatory response after traumatic brain injury: Implications for cellular therapy. Surgery. 2008;144(5):803-13.

29. Wang G, Zhang J, Hu X, Zhang L, Mao L, Jiang X, et al. Microglia/macrophage polarization dynamics in white matter after traumatic brain injury. Journal of Cerebral Blood Flow Metabolism: Official journal of the International Society of Cerebral Blood Flow Metabolism. 2013;33(12):1864-74.

30. Kigerl KA, Hall JC, Wang L, Mo X, Yu Z, Popovich PG. Gut dysbiosis impairs recovery after spinal cord injury. J Exp Med. 2016;213(12):2603-20.

31. Ritzel RM, Al Mamun A, Crapser J, Verma R, Patel AR, Knight BE, et al. CD200-CD200R1 inhibitory signaling prevents spontaneous bacterial infection and promotes resolution of neuroinflammation and recovery after stroke. J Neuroinflammation. 2019;16(1):40. 
32. He B, Hoang TK, Wang T, Ferris M, Taylor CM, Tian X, et al. Resetting microbiota by Lactobacillus reuteri inhibits $T$ reg deficiency-induced autoimmunity via adenosine A2A receptors. J Exp Med. 2017;214(1):107-23.

33. Kwon HK, Kim GC, Kim Y, Hwang W, Jash A, Sahoo A, et al. Amelioration of experimental autoimmune encephalomyelitis by probiotic mixture is mediated by a shift in $\mathrm{T}$ helper cell immune response. Clin Immunol. 2013;146(3):217-27.

34. Katzenberger RJ, Ganetzky B, Wassarman DA. The gut reaction to traumatic brain injury. Fly. 2015;9(2):68-74.

35. Winek K, Engel O, Koduah P, Heimesaat MM, Fischer A, Bereswill S, et al. Depletion of Cultivatable Gut Microbiota by Broad-Spectrum Antibiotic Pretreatment Worsens Outcome After Murine Stroke. Stroke. 2016;47(5):1354-63.

36. Manichanh C, Reeder J, Gibert P, Varela E, Llopis M, Antolin M, et al. Reshaping the gut microbiome with bacterial transplantation and antibiotic intake. Genome Res. 2010;20(10):1411-9.

37. Schaar KL, Brenneman MM, Savitz SI. Functional assessments in the rodent stroke model. Exp Transl Stroke Med. 2010;2(1):13.

38. Faden Al, Loane DJ. Chronic neurodegeneration after traumatic brain injury: Alzheimer disease, chronic traumatic encephalopathy, or persistent neuroinflammation? Neurotherapeutics. 2015;12(1):143-50.

39. Loane DJ, Kumar A, Stoica BA, Cabatbat R, Faden Al. Progressive neurodegeneration after experimental brain trauma: association with chronic microglial activation. J Neuropathol Exp Neurol. 2014;73(1):14-29.

40. Loane DJ, Stoica BA, Faden Al. Neuroprotection for traumatic brain injury. Handb Clin Neurol. 2015;127:343-66.

41. Nicholson SE, Merrill D, Zhu C, Burmeister DM, Zou Y, Lai Z, et al. Polytrauma independent of therapeutic intervention alters the gastrointestinal microbiome. Am J Surg. 2018;216(4):699-705.

42. Nicholson SE, Burmeister DM, Johnson TR, Zou Y, Lai Z, Scroggins S, et al. A prospective study in severely injured patients reveals an altered gut microbiome is associated with transfusion volume. $J$ Trauma Acute Care Surg. 2019;86(4):573-82.

43. Howard BM, Kornblith LZ, Christie SA, Conroy AS, Nelson MF, Campion EM, et al. Characterizing the gut microbiome in trauma: significant changes in microbial diversity occur early after severe injury. Trauma Surg Acute Care Open. 2017;2(1):e000108.

44. Taylor BE, McClave SA, Martindale RG, Warren MM, Johnson DR, Braunschweig C, et al. Guidelines for the Provision and Assessment of Nutrition Support Therapy in the Adult Critically III Patient: Society of Critical Care Medicine (SCCM) and American Society for Parenteral and Enteral Nutrition (A.S.P.E.N.). Crit Care Med. 2016;44(2):390-438.

\section{Supplemental Data}




\section{Figures}
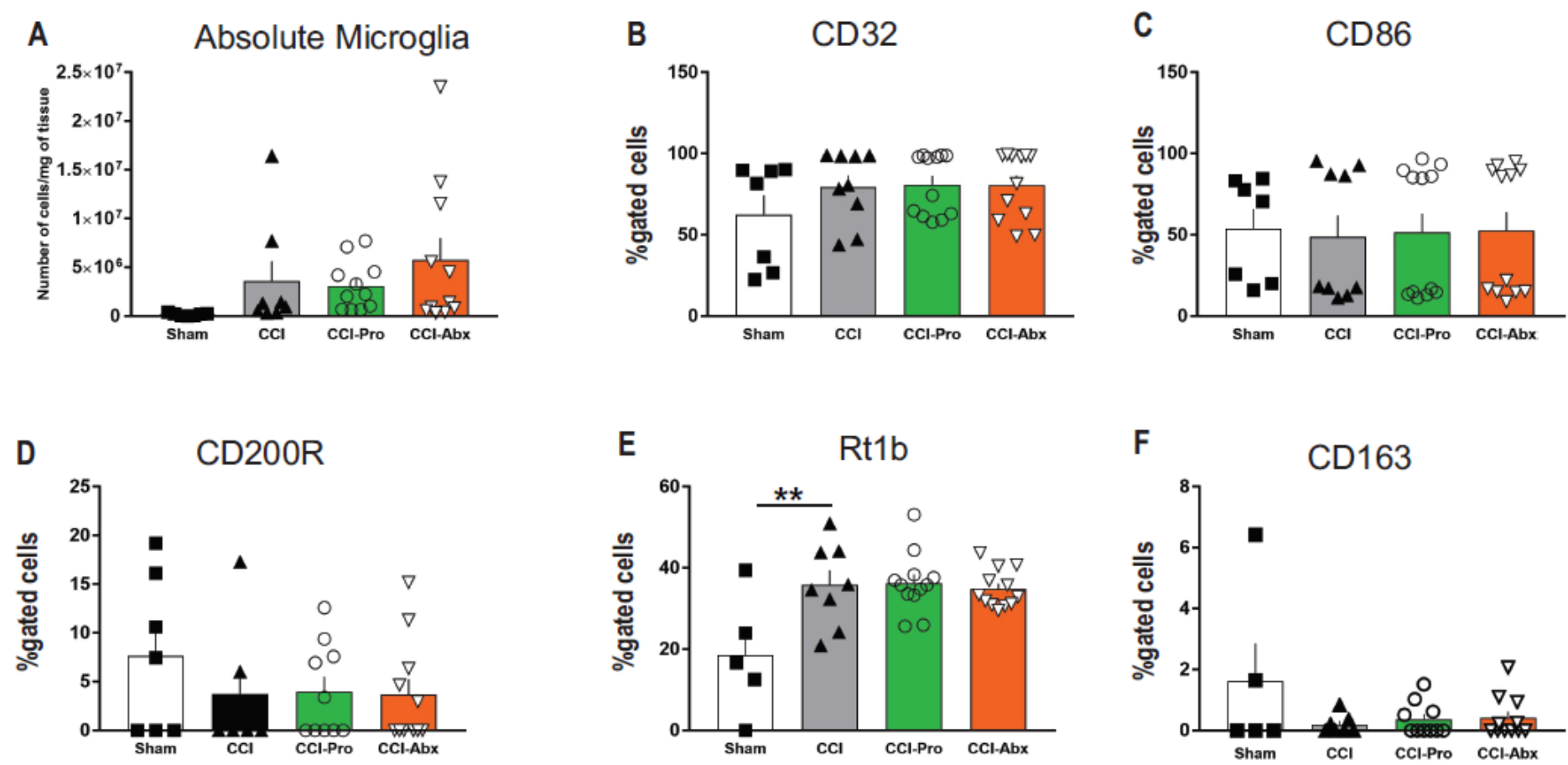

Figure 1

Microglia immunophenotyping analysis of ipsilateral (injured) hemispheres between experimental groups at 72 hours after injury: sham, injured animals without any treatments $(\mathrm{CCl})$, injured animals receiving probiotics (CCl-Pro), and injured animals receiving antibiotics (CCI-Abx) Seventy two hours after CCl, probiotic and antibiotic treatment do not affect pro- or anti-inflammatory surface markers. a. Absolute microglia per hemisphere calculated based on triplet anchor gating depicted. Values demonstrate number of microglia per milligram of tissue processed during cell isolation. b-c. Percent gated cells positive for CD32 and CD 86 on microglia cells, often correlated with M1 phenotype. d-f. Percent gated cells positive for CD200r, RT1b, and CD163 on microglia cells, demonstrating M2 phenotypic cells. Data are mean +/SEM, One-Way ANOVA, Dunnett's post-hoc test: $* *=p \leq 0.01$. 
A

Absolute Microglia
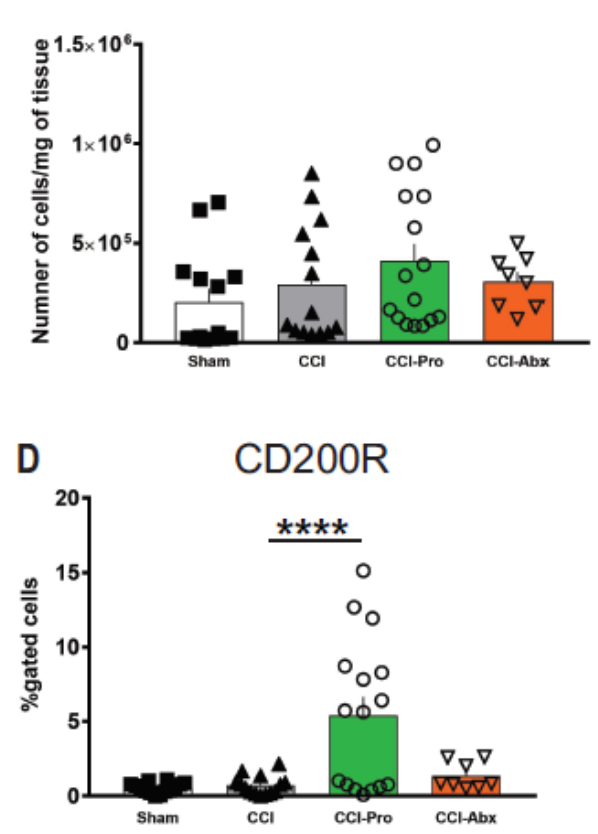

B

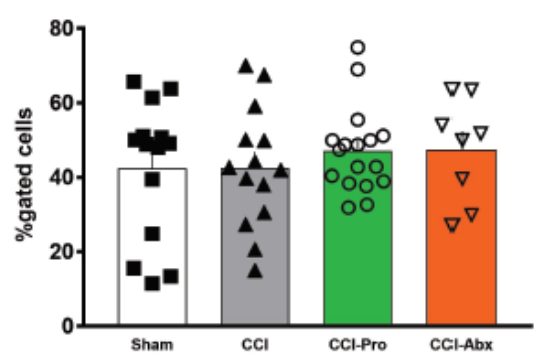

E

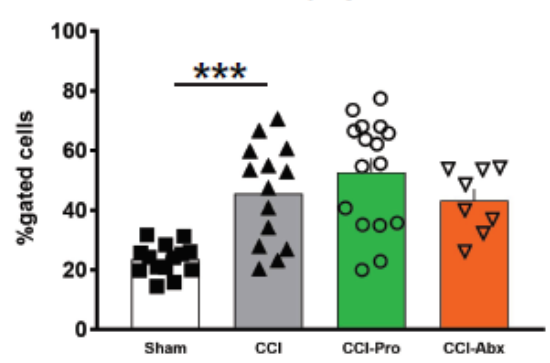

C

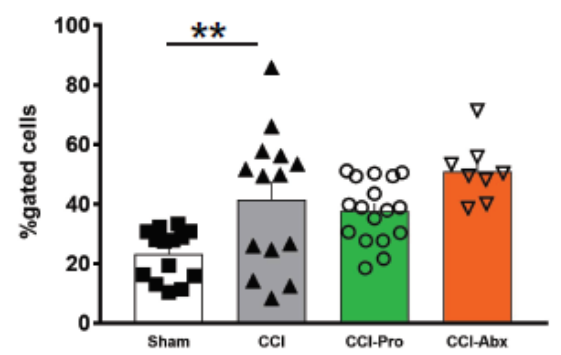

$\mathbf{F}$

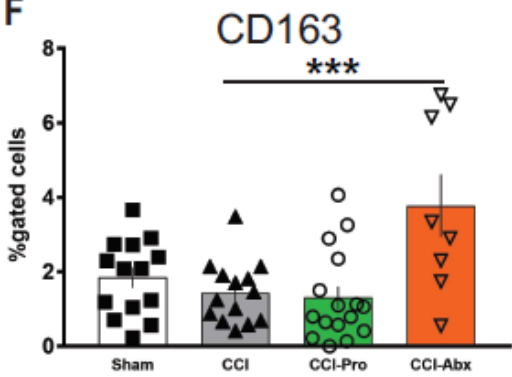

Figure 2

Microglia immunophenotyping analysis of ipsilateral (injured) hemispheres between experimental groups at 21 days after injury: sham, injured animals without any treatments $(\mathrm{CCl})$, injured animals receiving probiotics (CCl-Pro), and injured animals receiving antibiotics (CCl-Abx). Twenty one days after $\mathrm{CCl}$, probiotic and antibiotic treatment increase anti-inflammatory surface markers. a. Absolute microglia per hemisphere calculated based on triplet anchor gating depicted in Fig. 1. Values demonstrates number of microglia per milligram of tissue processed during cell isolation. b-c. Percent gated cells positive for CD 32 and CD86 on microglia cells, associated with M1 phenotype. d-f. Flow analysis of percent gated cells positive for CD200r, RT1b, and CD163 on microglia, demonstrating M2 phenotypic cells. Data are mean

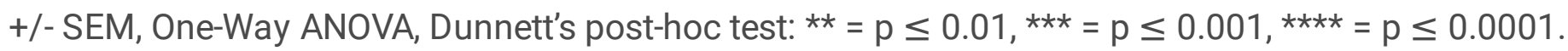


A

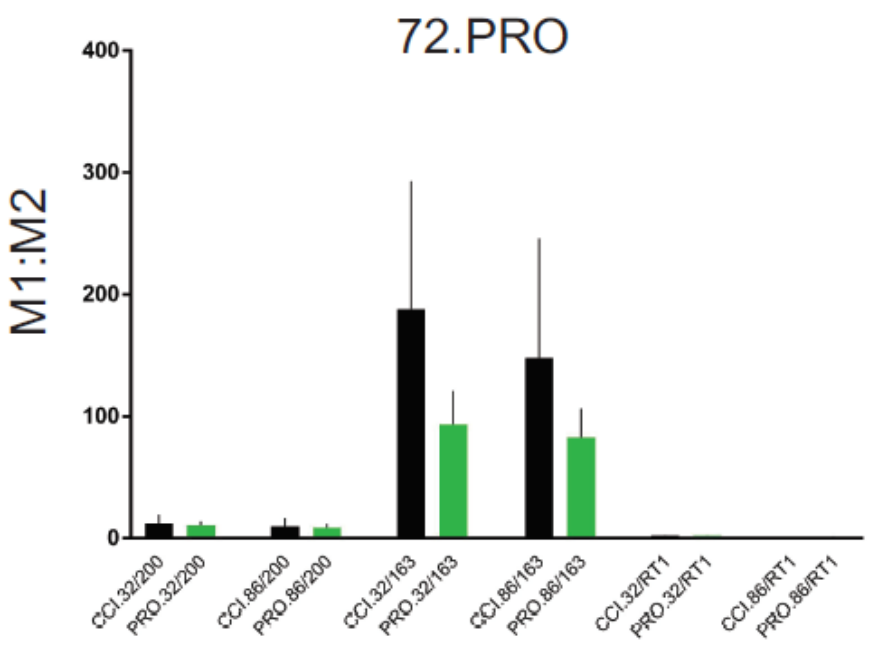

C
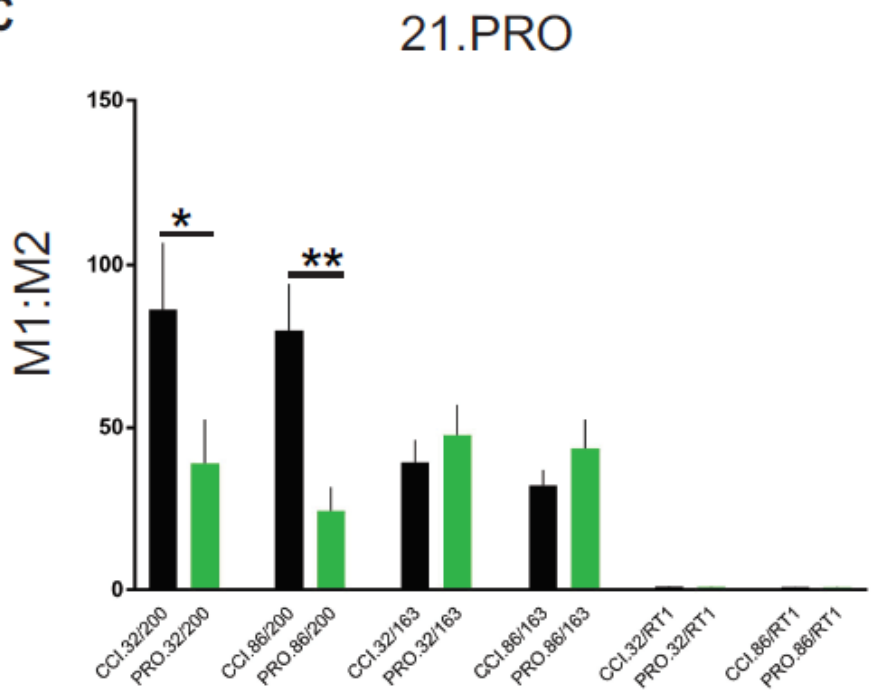

B

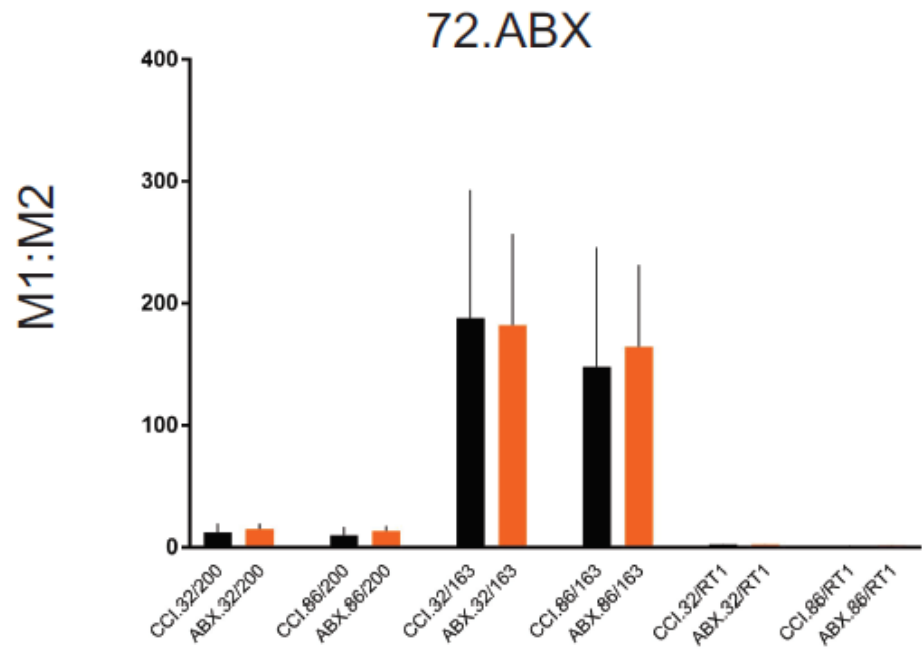

D

21. $A B X$

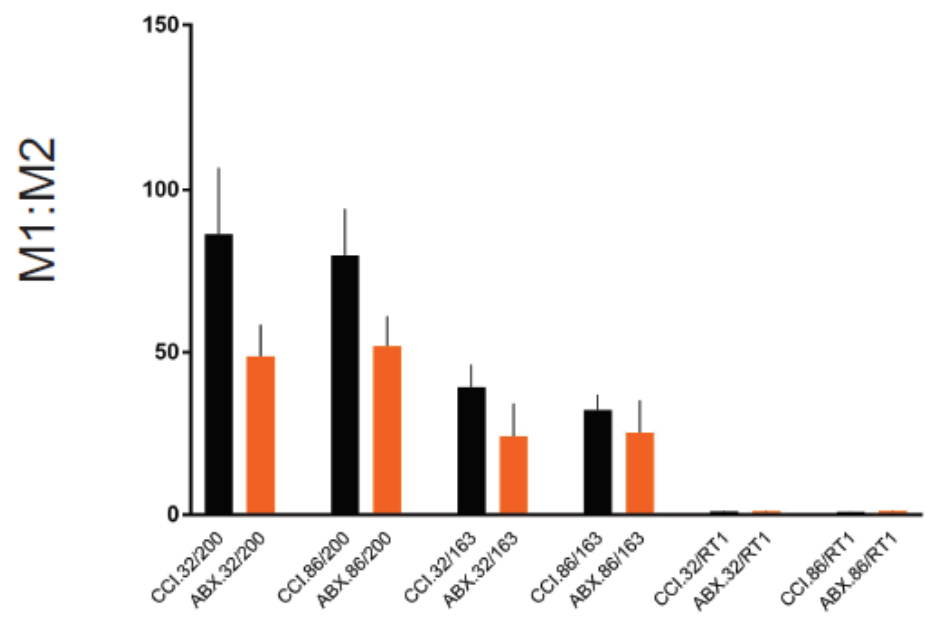

\section{Figure 3}

Relative ratios of $\mathrm{M} 1 / \mathrm{M} 2$ ratios transposed between antibiotic and probiotic treatment groups. Twenty one days after $\mathrm{CCl}$, probiotic treatment attenuate $\mathrm{M} 1 \mathrm{M} 2$. Higher $\mathrm{M} 1 / \mathrm{M} 2$ ratios correlate with microglia polarization favoring a pro-inflammatory phenotype. a-b. M1:M2 ratios at 72 hours demonstrate no significant differences. c-d. M1:M2 ratios at 21 days demonstrate probiotic treatment significant decreases neuroinflammation when compared to $\mathrm{CCl}$ alone. Data are mean +/- SEM, Student's T-Test: * = $p \leq 0.05, * \star=p \leq 0.001$. 
A Natural Killer Cells(CD161)

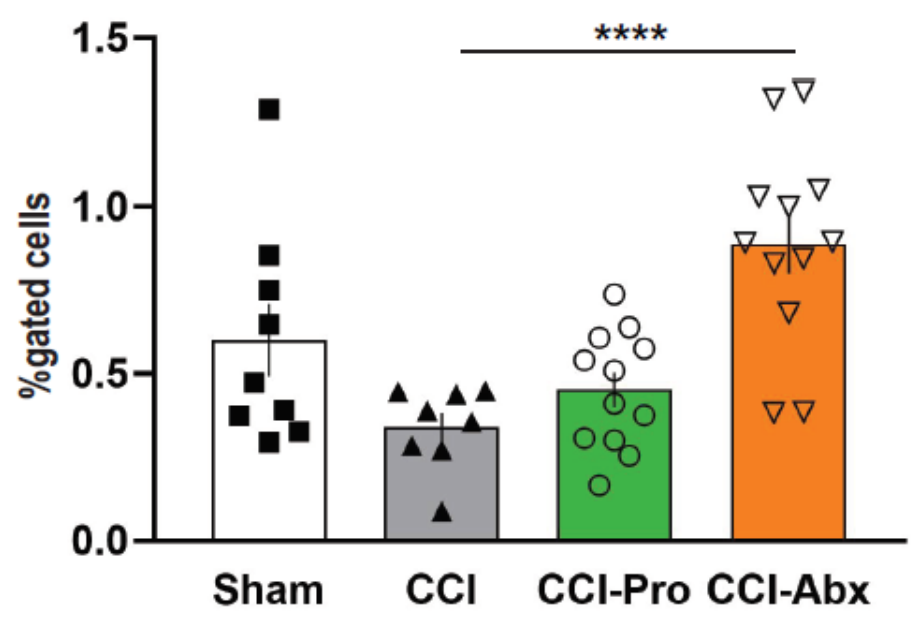

B

Monocytes (CD172a+)

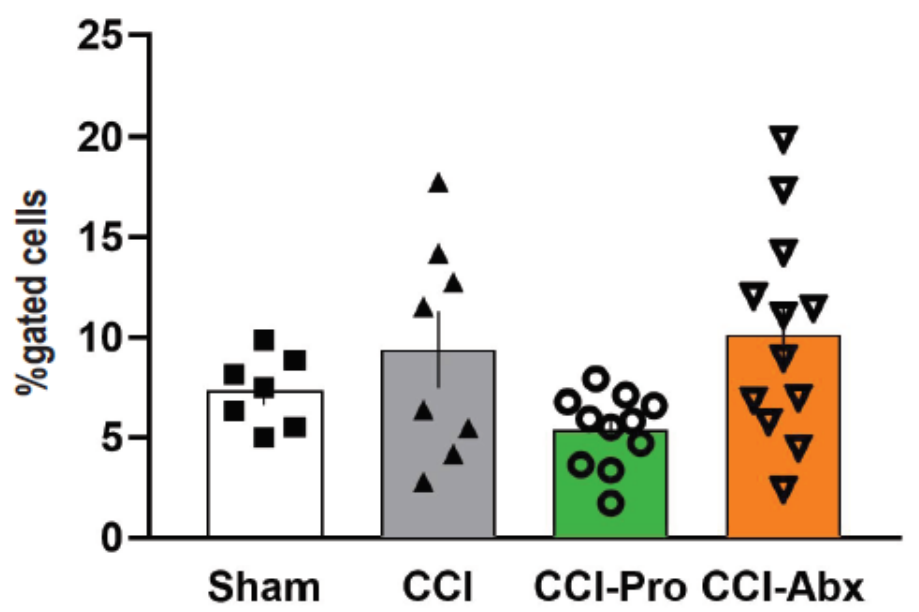

\section{Figure 4}

Immunophenotyping of isolated cells from intestinal Peyer's Patches at 72 hours after injury: sham ( $\mathrm{n=7}$ ), injured animals without any treatments $(\mathrm{CCl}, \mathrm{n}=8)$, injured animals receiving probiotics $(\mathrm{CCl}-\mathrm{PrO}, \mathrm{n}=12)$, and injured animals receiving antibiotics (CCl-Abx, $n=12)$. a. Seventy-two hours after $\mathrm{CCl}$, antibiotic treatment increases natural killer cells in Peyer's Patches. b. Neither antibiotic or probiotic treatment affected monocytes in the Peyer's patches, however there was an overall significant as measured by One-

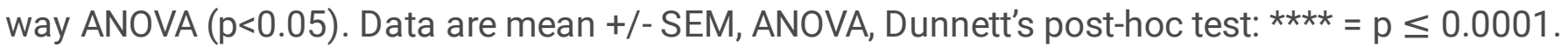



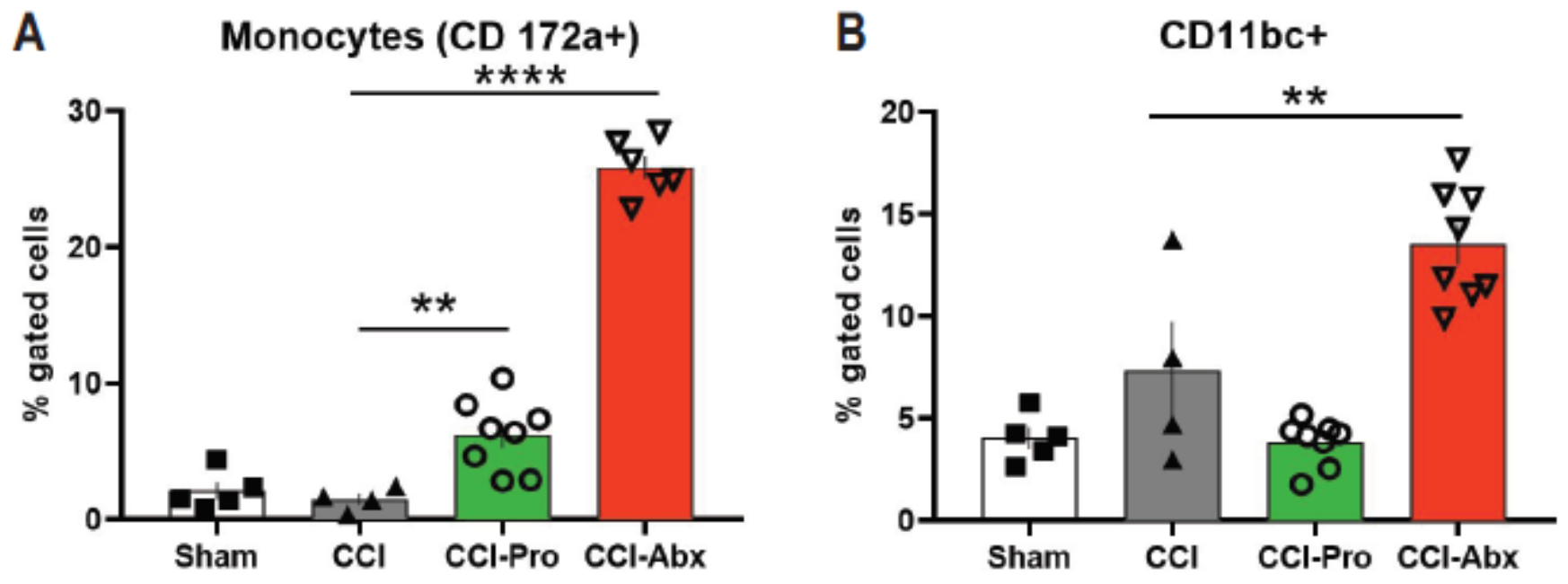

C Natural Killer Cells (CD161+)

D
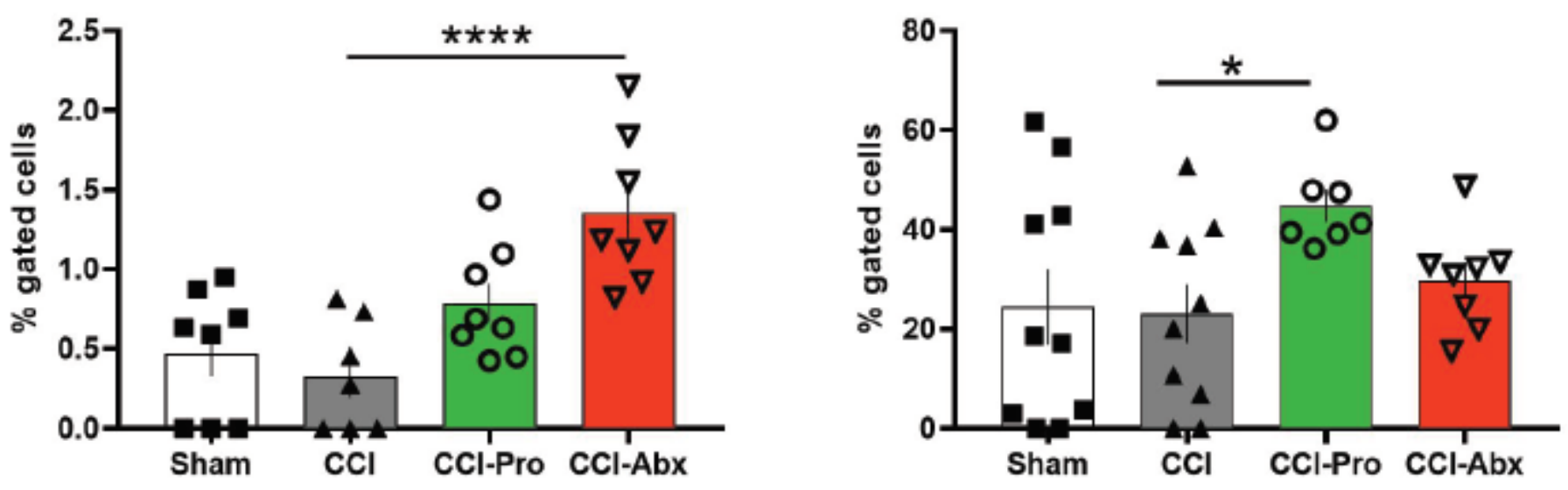

Figure 5

Immunophenotyping of isolated cells from intestinal Peyer's Patches at 21 days after injury: sham $(n=8)$, injured animals without any treatments $(\mathrm{CCl}, \mathrm{n}=8)$, injured animals receiving probiotics (CCI-Pro, $n=8)$, and injured animals receiving antibiotics $(\mathrm{CCl}-\mathrm{Abx}, \mathrm{n}=8)$. Cells are demonstrated as percent gated cells to allow for comparison between experimental groups. a-d. Twenty-one days after TBI, antibiotic and probiotic treatments increase monocytes, CD11bc+, natural killer cells, and CD3+ cells in Peyer's patches. Data are mean +/-SEM, ANOVA, Dunnett's post-hoc test: ${ }^{*}=p \leq 0.05, * \star=p \leq 0.01, * \star \star \star * p \leq 0.0001$. 


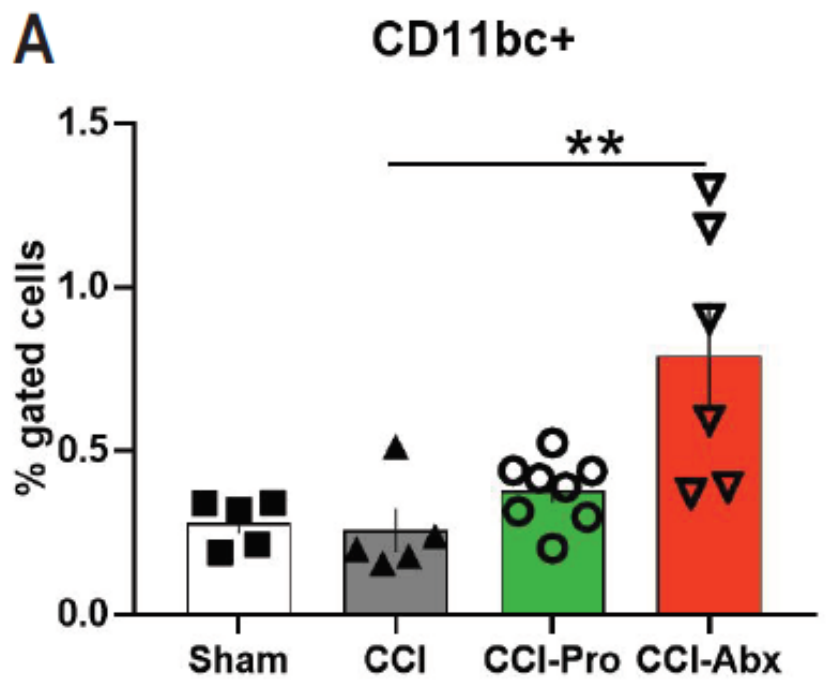

B Natural Killer Cells (CD161+)

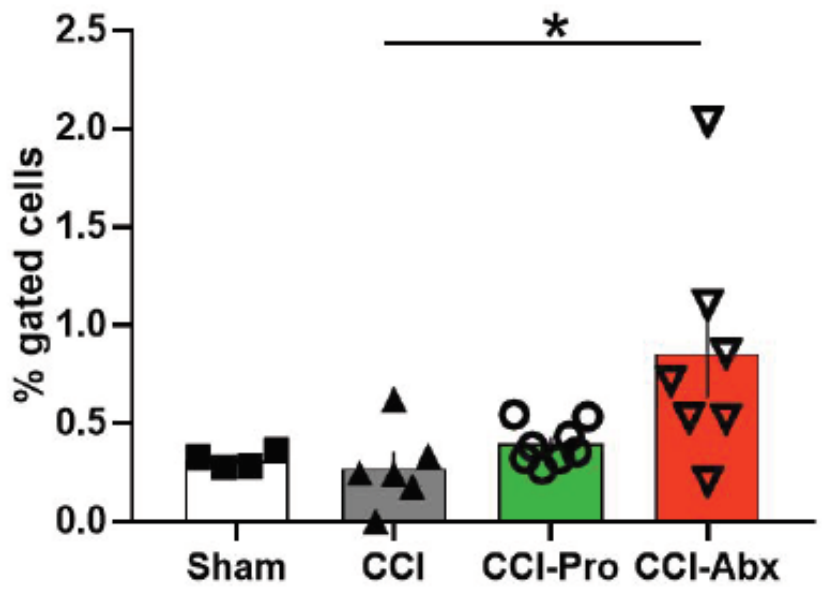

Figure 6

Immunophenotyping of isolated cells from mesenteric lymph nodes at 21 days after injury: sham $(n=8)$, injured animals without any treatments $(\mathrm{CCl}, \mathrm{n}=8)$, injured animals receiving probiotics (CCl-Pro, $n=8)$, and injured animals receiving antibiotics $(\mathrm{CCl}-\mathrm{Abx}, \mathrm{n}=8)$. Cells are demonstrated as percent gated cells to allow for comparison between experimental groups. a-b. At 21 days post injury, CD11bc+ and CD161 activation is amplified by antibiotics, demonstrating a pro-inflammatory response in the mesenteric lymph nodes. Data are mean +/- SEM, ANOVA, Dunnett's post-hoc test: * $=p \leq 0.05,{ }^{\star *}$. 

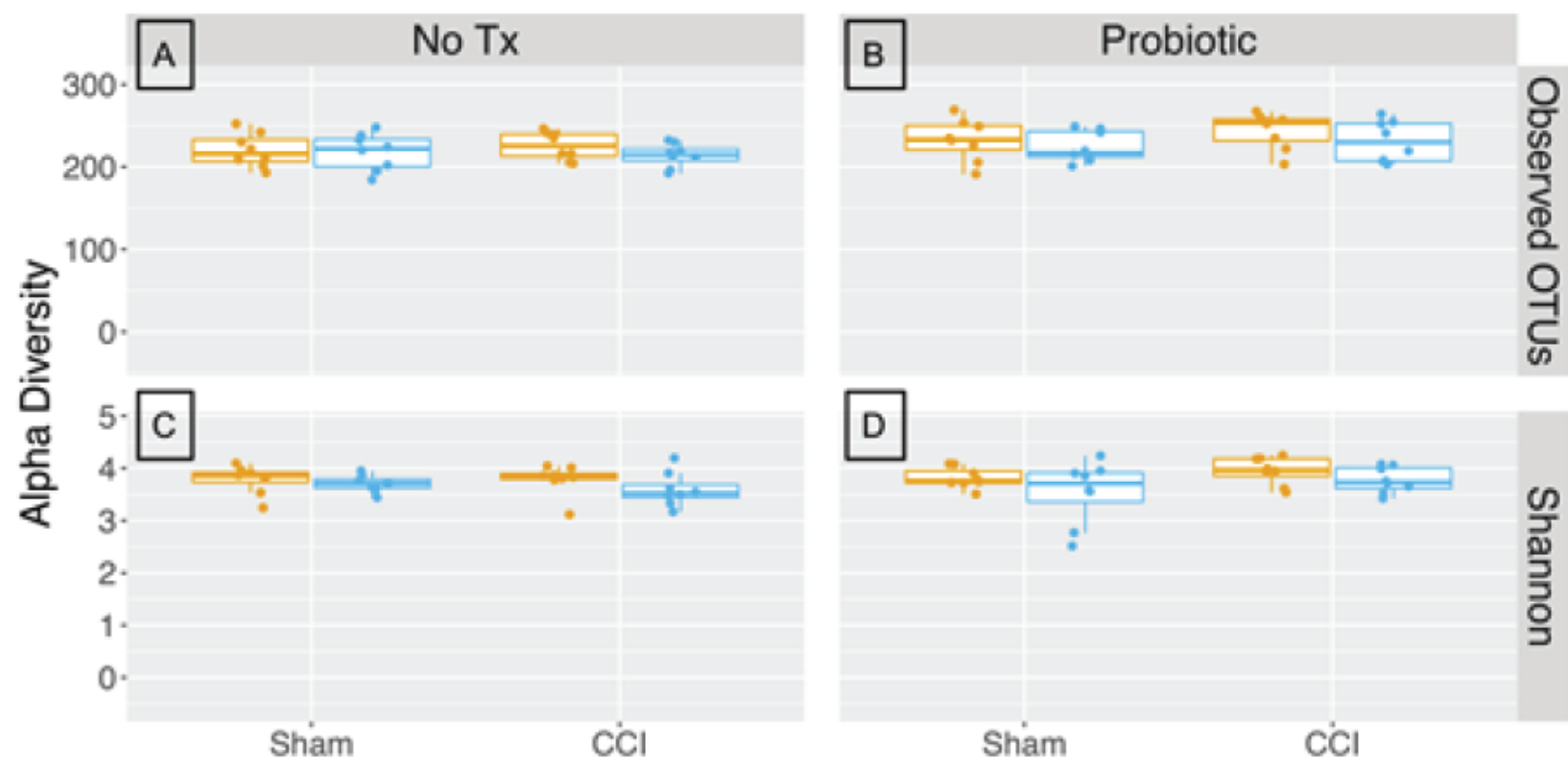

'Time Point"

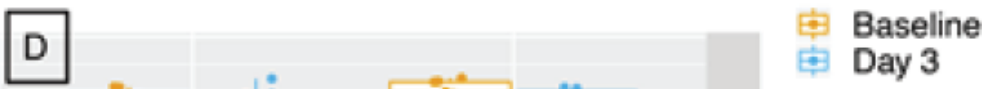

Sham

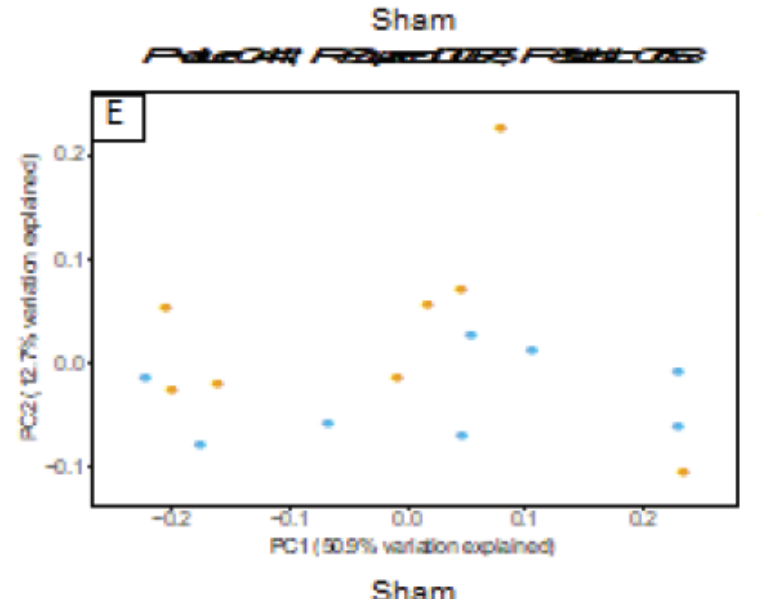

Sham

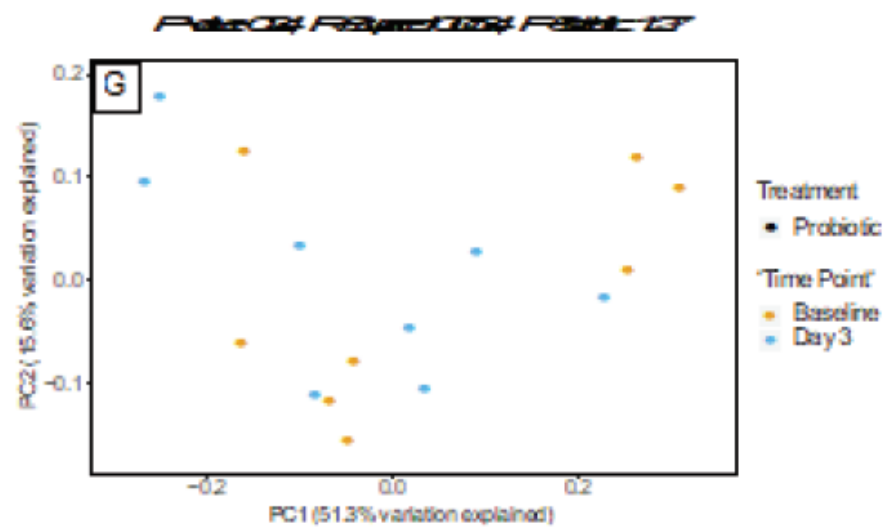

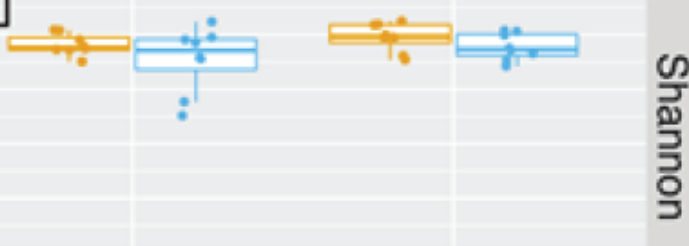

Sham $\quad \mathrm{CCl}$

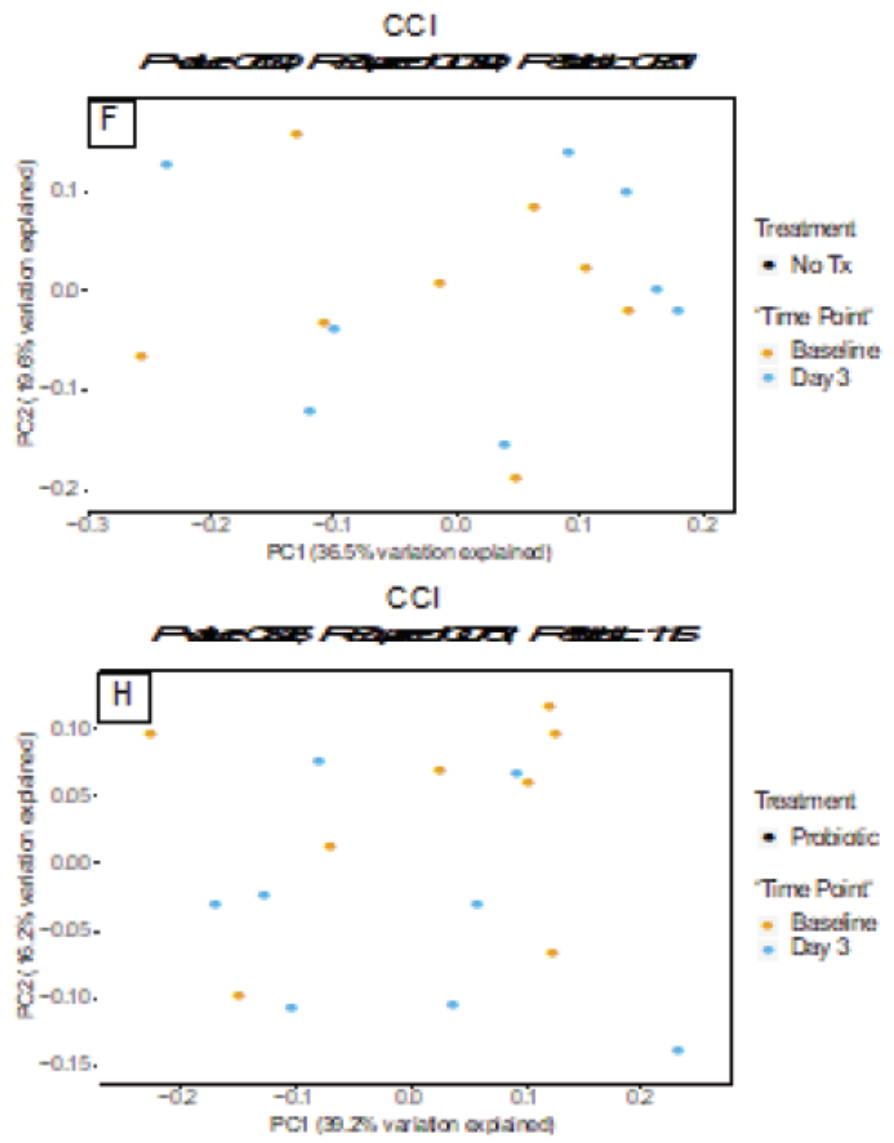

Figure 7

There is no change in the alpha $(\mathbb{\nabla})$ diversity between Sham $(n=8)$ and severely injured $(C C l, n=8)$ animals over a time course of 3 days. a-d. Fig. a and b display observed organized taxonomic units (OTUs). Fig. c and d display the $\nabla$ diversity as the Shannon indices. Fig.a and $\mathrm{c}$ show the $\otimes$ diversity for animals that received no probiotics (No Tx) and the two experimental groups are further stratified into two timepoints: baseline (orange) and post-injury day 3 (blue). Fig. $b$ and d show animals that were treated with probiotic Lactobacillus reuteri (LR) and are also stratified into two timepoints. Baseline samples were obtained 
prior to injury and/or treatment. Day 3 samples were obtained 3 days after $\mathrm{CCl}$. There were no significant differences found between groups using the Kruskal-Wallis test. e-h. There is no change in the beta ( $\beta$ ) diversity between Sham $(n=8)$ and severely injured $(C C l, n=8)$ animals over a time course of 3 days. Fig. e represents Sham animals that did not receive probiotics (No Tx) as a time course over 3 days. Baseline fecal samples were collected prior to injury (orange). Day 3 fecal samples were obtained 3 days after $\mathrm{CCI}$ (blue). Fig. f represents injured animals $(\mathrm{CCl})$ that did not receive probiotics and displays the progression of the $\beta$ diversity over 3 days. There was no change in the $\beta$ diversity of the microbiome with injury alone. Fig. $g$ represents Sham animals that received probiotics for 3 days after injury. Baseline sample (orange) was collected prior to injury and treatment with probiotics. There was no change in the $\beta$ diversity of the microbiome with probiotics alone. The $\beta$ diversity displayed is a measure of weighted UniFrac distances; PCoA plots utilize Monte Carlo permutation tests to estimate $p$-values. 


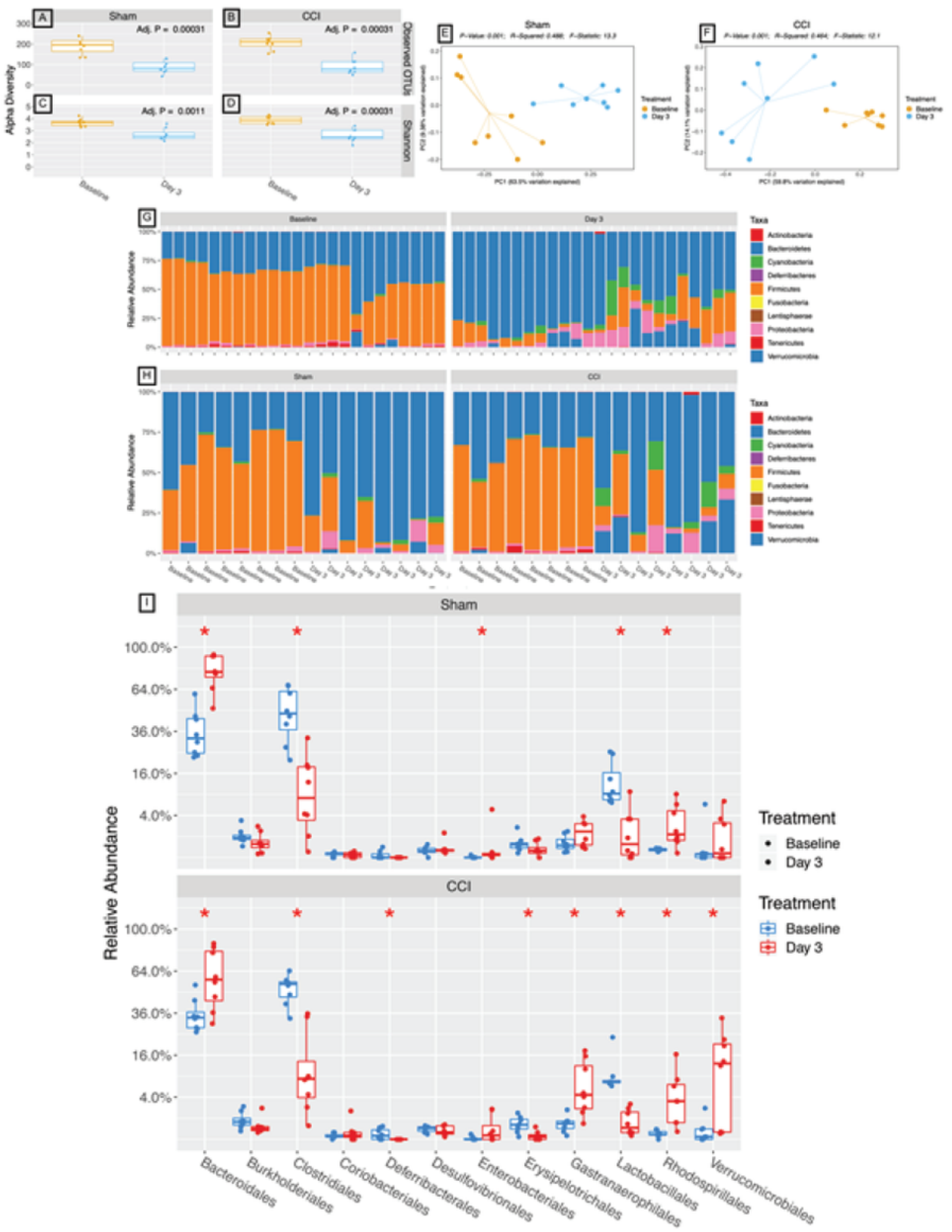

\section{Figure 8}

a-d. There is significant reduction of alpha $(\mathbb{\nabla})$ diversity over a time course of 3 days after being treated with broad spectrum antibiotics. Animals received antibiotics for 7 days prior to becoming injured $(n=8)$. e-f. There is a significant shift in the $(\beta)$ diversity within Sham $(n=8)$ and $\mathrm{CCl}$ animals $(n=8)$ after treatment with antibiotics. There was a significant shift in the $\beta$ diversity of the microbiome with treatment of antibiotics. The $\beta$ diversity displayed is a measure of weighted UniFrac distances; PCoA 
plots utilize Monte Carlo permutation tests to estimate p-values. Statistical analyses utilized KruskalWallis tests. g-h. Treatment with antibiotics caused a significant change in the relative abundance based on the most abundant phyla isolated from fecal samples. Fig. $g$ represent the entire cohort of animals that received antibiotics $(n=16)$. Fig. $h$ further stratify the data into Sham $(C, n=8)$ and $C C I(D, n=8)$ and show the progression of their microbiome from baseline to post-injury day 3 , while receiving antibiotic treatment. i. There are significant differences between relative abundances between experimental groups when stratified into taxonomic order. The left sided plot demonstrates Sham animals, and the right sided plot demonstrates injured $(\mathrm{CCl})$ animals. Within the Sham animals, there are multiple significant differences between the baseline sample and day 3 sample. However, while the same trend is seen within the $\mathrm{CCl}$ animals, there are several more significant changes in the microbiome profile of these animals when compared to Sham animals. Statistical analysis was done utilizing Mann-Whitney tests. $(*=p \leq$ $0.05)$. 